Cahiers de recherches médiévales

Journal of medieval studies

14 | 2007

L'héritage de Chrétien de Troyes

\title{
Girart d'Amiens
}

Un auteur et une œuvre à la fin du XII ${ }^{\mathrm{e}}$ siècle

Silvère Menegaldo

\section{OpenEdition}

Journals

Édition électronique

URL : https://journals.openedition.org/crm/2673

DOI : $10.4000 / \mathrm{crm} .2673$

ISSN : 1955-2424

Éditeur

Honoré Champion

Édition imprimée

Date de publication : 15 décembre 2007

Pagination : 145-151

ISSN : 1272-9752

Référence électronique

Silvère Menegaldo, "Girart d'Amiens », Cahiers de recherches médiévales [En ligne], 14 | 2007, mis en ligne le 15 décembre 2010, consulté le 15 décembre 2022. URL : http://journals.openedition.org/crm/ 2673 ; DOI : https://doi.org/10.4000/crm.2673 


\section{酮RM}

\section{Girart d'Amiens : un auteur et une ouvre à la fin du XIII ${ }^{\mathrm{e}}$ siècle}

Qu'est-ce que Girart d'Amiens aujourd'hui? Un nom attaché à une œuvre, une œuvre attachée à un nom - ce qui n'est pas si négligeable somme toute au Moyen Âge, où l'anonymat des textes et les problèmes d'attribution sont monnaie courante.

À propos de l'œuvre, depuis peu on dispose enfin d'une édition moderne de toutes les compositions de Girart d'Amiens, après la publication de L'Istoire le roy Charlemaine par D. Métraux en 2004. Et si la qualité littéraire de ces textes a pu être mise en doute, en particulier par Gaston Paris et Léon Gautier, si de fait il n'est peutêtre pas très aisé d'attirer sur eux un regard bienveillant, car il ne s'agit pas non plus d'immortels chefs-d'œuvre, reste que ces textes n'en présentent pas moins l'intérêt de constituer un ensemble explicitement rattaché au même auteur, autrement dit une œuvre. De cette œuvre, rappelons brièvement les trois (longs) pans, dans l'ordre probable de leur composition : d'abord Escanor, roman arthurien de près de 26000 vers, que son dernier éditeur, R. Trachsler ${ }^{1}$, propose de situer vers 1280 ; Meliacin, ensuite, qui relève plutôt du roman d'amour et d'aventure d'inspiration orientale, long de plus de 19000 vers, aurait été composé selon A. Saly ${ }^{2}$ vers 1285 ; enfin Girart a rimé L'Istoire le roy Charlemaine, poème épique comptant plus de 23000 vers, au début du XIV ${ }^{\mathrm{e}}$ siècle, soit vers 1301-1303 comme le propose D. Métraux ${ }^{3}$, soit vers 1303-1306 d'après A. Saly ${ }^{4}$.

Dans chacun de ces trois textes, l'auteur se nomme explicitement: Gerardin (v. 4) pour la première fois dans le prologue d'Escanor, puis Gerart (v. 50), et dans l'épilogue Girardin d'Amiens (v. 25900) et Girart (v. 25910); de nouveau dans l'épilogue de Meliacin il use de l'hypocoristique Girardin d'Amiens (v. 19131); enfin il se désigne comme Girar, Girart ou Gyrart (v. 73) d'Amiens (v. 14, 19205, 19219, 23329) dans L'Istoire le roy Charlemaine. Malheureusement, au tournant des $\mathrm{XIII}^{\mathrm{e}}$ et $\mathrm{XIV}^{\mathrm{e}}$ siècles, le nom de Girart n'est pas des plus rares du côté d'Amiens et rien ne permet, en dépit des tentatives faites en ce sens par A. Saly ${ }^{5}$, d'identifier le nom de l'auteur de ces textes avec celui d'un personnage réel. Les dédicaces de deux de ses œuvres permettent néanmoins de situer et de dater avec quelque précision l'activité littéraire de Girart, et surtout de montrer l'auteur en contact avec la plus haute noblesse. En effet la dédicace initiale d'Escanor renvoie sans doute

\footnotetext{
${ }^{1}$ Escanor, éd. R. Trachsler, Genève, Droz (Textes Littéraires Français 449), 1994, tome un, p. 27-29.

2 A. Saly, «Les destinataires du roman de Meliacin», Travaux de linguistique et de littérature, 19, 2, 1981, p. 7-16.

${ }^{3}$ L'Istoire le roy Charlemaine, éd. D. Métraux, Lewiston, Edwin Mellen, 2004, tome un, p. xxiv-xxvii.

4 A. Saly, "La date du Charlemagne de Girart d'Amiens", Au carrefour des routes d'Europe : la chanson de geste, Senefiance, 21, 1987, p. 975-981.

${ }^{5}$ A. Saly, Édition critique du "Meliacin» de Girart d'Amiens, accompagnée d'une étude historique et littéraire, Thèse de Doctorat d'État, Université de Paris IV, 1977, p. 64-70.
}

Cahiers de Recherches Médiévales, 14, 2007 
possible à Aliénor de Castille, reine d'Angleterre et épouse d'Édouard ${ }^{\text {er }}$; et de son côté L'Istoire le roy Charlemaine est clairement dédiée à Charles de Valois, frère du roi de France Philippe IV le Bel. Quant au destinataire de Meliacin, il est plus difficile à déterminer; cependant, au terme d'un examen de la grande miniature initiale que comportent deux des manuscrits du roman, A. Saly en conclut que si l'œuvre a dû être commanditée par Gaucher de Châtillon, connétable de Philippe le Bel, le sujet en a probablement été soufflé par Blanche de France, de même d'ailleurs que pour le Cleomadés d'Adenet le Roi - roman qui constitue, rappelonsle, une composition jumelle du Meliacin, les deux auteurs ayant apparemment été mis en concurrence pour relater à leur façon la même histoire?

Girart d'Amiens entre donc dans le cercle relativement restreint, aux XII ${ }^{\mathrm{e}}$ et $\mathrm{XIII}^{\mathrm{e}}$ siècles, des auteurs dotés d'une œuvre nettement constituée. À l'intérieur de ce cercle, il apparaît se situer dans une position assez comparable à celle d'un Chrétien de Troyes ou d'un Rutebeuf par exemple, c'est-à-dire un simple nom d'auteur, non identifié, mais associé à une œuvre stable; soit dans une position intermédiaire entre un Wace, un Philippe de Rémi ou un Adenet le Roi d'un côté, c'est-à-dire des auteurs qui signent leurs œuvres et dont l'existence se trouve en quelque sorte confirmée par des documents non littéraires (ce qui, à dire vrai, n'avance pas forcément la critique à grand-chose), et un Jean Renart de l'autre, dont le nom n'est attesté qu'à une reprise dans l'épilogue du Lai de l'Ombre, et l'œuvre issue d'une reconstitution de la critique moderne. On notera en outre, à la suite d'A. Corbellari et D. Métraux, un autre point susceptible, si l'on peut dire, d'asseoir la position d'auteur de Girart, c'est la grande stabilité textuelle de son œuvre ${ }^{8}$, même si elle ne peut être jugée qu'à l'aune d'un petit nombre de manuscrits - on en compte sept à peu près complets, dont par ailleurs aucun, il faut le préciser, ne contient plus d'un texte de l'auteur.

En somme Girart d'Amiens bénéficie à la fin du XIII ${ }^{\mathrm{e}}$ siècle d'un statut d'auteur relativement affirmé, bien plus qu'un Jean Renart par exemple. L'auteur et son œuvre n'ont pourtant donné lieu, à la différence d'un Jean Renart justement, et sans parler d'un Chrétien de Troyes ou d'un Rutebeuf, à aucune étude d'ensemble depuis la thèse de 1958 de G.J. Brault. Il est vrai que dans le domaine médiéval l'ensemble constitué par un auteur et son œuvre tend parfois à être oblitéré par la critique, trop habituée peut-être à raisonner en termes d'anonymat et de pseudographie, inévitablement marquée par les séquelles du structuralisme et de la «mort de l'auteur $»^{10}$, pour ne rien dire de certains effets de mode. Dans le cas particulier de

\footnotetext{
${ }^{6}$ A. Saly, « Les destinataires du roman de Meliacin ».

${ }^{7}$ Voir sur ce point particulier A. Saly, «Écrivains mystificateurs : le cas d'Adenet le Roi et de Girart d'Amiens », Figures de l'écrivain au Moyen Âge, éd. D. Buschinger, Göppingen, Kümmerle, 1991, p. 245-253.

${ }^{8}$ « Ce qui atteste finalement avec le plus d'évidence le statut d'auteur de Girart, c'est la remarquable stabilité textuelle des copies de ses œuvres", A. Corbellari et D. Métraux, «Sacré Charlemagne ! », Études de lettres, 1999, 2, p. 118.

${ }^{9}$ G.J. Brault, A Study of the Works of Girart d'Amiens, Ph.D., University of Pennsylvania, 1958.

${ }^{10}$ Pour reprendre le titre d'un article fameux de R. Barthes. On observe cependant depuis quelque temps un mouvement inverse de résurrection de l'auteur, le concept retrouvant son
} 
Girart, néanmoins, il est possible que la véritable difficulté réside plutôt dans cette «personnalité » littéraire que, selon A. Corbellari et D. Métraux, «on chercherait en vain dans les trois œuvres-fleuves de Girart ${ }^{11}$. De fait le succès critique de Chrétien de Troyes ou de Jean Renart peut certainement s'expliquer dans la mesure où leur œuvre - attestée ou reconstituée - fait effectivement œuvre, c'est-à-dire présente un ensemble suffisamment significatif de centres d'intérêt et de points communs, en somme une personnalité littéraire suffisamment marquée pour justifier une approche d'ensemble ; ce qui va beaucoup moins de soi pour Girart, dont le succès semble-t-il assez limité dès le Moyen Âge, si l'on en juge d'après le nombre de manuscrits conservés, n'est peut-être pas l'entier effet du hasard.

Probablement parce qu'il a édité toute son œuvre et qu'il l'a longuement fréquentée, A. Henry a pu s'essayer dans sa "Biographie d'Adenet le Roi » ${ }^{12}$ à atteindre le "personnage intérieur» de l'auteur, à en dresser un portrait à la fois psychologique et littéraire qui montre à l'œuvre chez Adenet «la morale du ménestrel de cour» et fait de ce que le critique appelle son " conformisme souriant» un de ses traits caractéristiques ${ }^{13}$.

$\mathrm{Ne}$ serait-il pas alors envisageable, au moins sur un plan plus strictement littéraire, de se prêter au même exercice avec Girart d'Amiens? En admettant de considérer Escanor, Meliacin et L'Istoire le roy Charlemaine comme un ensemble solidaire, comme une œuvre attachée à un auteur - en dépit de D. Métraux, selon qui «c'est en vain que l'on chercherait une ressemblance quelconque entre les trois poèmes de Girart $»^{14}-$, ne pourrait-on essayer de définir quelques traits de la personnalité littéraire de Girart, ou du moins de faire quelques propositions en ce sens, en relevant par exemple son goût pour une esthétique de la copia, qui touche d'ailleurs toutes les strates du texte (récit, descriptions, monologues et dialogues); pour la variation - certes un peu épuisée... - autour des genres canoniques que sont le roman arthurien, le roman d'aventure et la chanson de geste (ou la chronique); ou encore pour certains thèmes, comme l'amitié entre deux ennemis, ainsi que le suggère A. Saly? L'exercice - exigeant de scruter de près trois longs textes d'une

importance dans le domaine des études littéraires. Pour une mise au point sur cette question, voir par exemple A. Compagnon, Le Démon de la théorie, Paris, Seuil, 1998, p. 47-99.

${ }^{11}$ A. Corbellari et D. Métraux, art. cit., p. 117.

${ }^{12}$ A. Henry, Les Euvres d'Adenet le Roi, De Tempel, Bruges, tome un, 1951, p. 11-64.

${ }^{13}$ Ibid., p. 60-61 : «Faire l'éloge de la largesse, exalter les sentiments aristocratiques, vanter la fidélité au suzerain, dauber, en passant, le vilain, inviter à la courtoisie, chanter la vague justice en signalant parfois, du bout des doigts, les exactions des grands, prôner la bravoure, la protection du faible, le respect des femmes, dire le bien, taire le mal, n'ouvrir la bouche que pour les beaux dits et les douces paroles: voilà la morale d'Adenet, c'est la morale du ménestrel de cour ». «Pieux et reconnaissant, complaisant et serviable, esprit délicat et même raffiné, Adenet a toutes les qualités, un peu ternes mais plaisantes, qui créent la douceur enjouée et la sociabilité amène. Il aime la vie seigneuriale, les réunions de cour, les discussions sur l'héraldique et la chevalerie, la conversation des dames, la vie calme, la tiédeur des chambres closes aux tapisseries protectrices. Il goûte les fêtes et les réjouissances et il admire les bijoux étincelants, les joyaux de prix, les vêtements drapés aux chauds tissus chatoyants », etc.

${ }^{14}$ L'Istoire le roy Charlemaine, éd. cit., p. xii. 
écriture assez diffuse et dont on ne saurait exactement dire s'ils sont novateurs ou d'arrière-garde, à moins que ce ne soit les deux à la fois - n'est pas des plus aisés évidemment, et pourrait s'avérer décevant. Aussi nous ne faisons ici que suggérer de l'entreprendre, comme inciterons peut-être aussi à le faire les études qui suivent de D. de Carné, A. Saly ${ }^{15}$, A. Corbellari et D. Métraux, la première consacrée à Escanor, la seconde à Meliacin et les deux dernières à L'Istoire le roy Charlemaine - ou au moins pourront-elles contribuer à redonner un peu de lustre au rival négligé d'Adenet le Roi. D'ailleurs, en dépit peut-être d'un manque de personnalité littéraire, on reconnaîtra que les rapports d'émulation apparemment entretenus par Adenet le Roi et Girart d'Amiens autour de Cleomadés et de Meliacin suggèrent au moins chez Girart une forme relativement développée de conscience d'auteur, qui se sent capable en rivalisant avec un confrère d'imprimer sa propre marque à la matière brute du sujet procuré par ses nobles commanditaires. Ainsi, à défaut probablement d'être un grand auteur, au moins Girart d'Amiens avait-il conscience d'en être un, ce qui n'est déjà pas si mal.

Silvère Menegaldo Université d'Orléans

\footnotetext{
${ }^{15}$ A. Saly qui, il faut le préciser, s'efforce justement dans son étude de mettre en évidence la «personnalité propre » des deux auteurs dans sa comparaison de Meliacin et de Cleomadés.
} 